\title{
Managing operations in real-time
}

\author{
R. L. Saldanha, A. Frazõ, J. P. Martins \& E. Morgado \\ SISCOG, Sistemas Cognitivos SA Lisboa, Portugal
}

\begin{abstract}
Accurate knowledge about the state of the operation of a railway, together with decision-support tools to help solve the problems that occur during the operation, are key issues for the timely provision of services to the public. Modern communications enable real-time availability of information about the state of execution of the operations. Associated with real-time communication, new kinds of software products are emerging that provide effective support to railway dispatchers. The paper presents a real-time dispatching product developed by SISCOG that enables real-time monitoring of what is happening during the operation and provides optimizers to handle operational disruptions. Scenariobased functionality to address major traffic disruptions is under development. Systems based on this product are in use by several European railways.

Keywords: crew scheduling, crew planning, crew dispatching.
\end{abstract}

\section{Introduction}

The pressure for environmental-friendly transportation associated to the growing needs for mass transport and the possibility of competing with airlines in mediumrange routes are generating a railway expansion that had not been seen for almost one century. Alongside with this expansion, concerns for productivity and quality of service are leading railway companies to introduce new management styles to improve the results of the business. In particular, new tools are being searched to improve the use of human, material, and track resources, as well as to increase the performance of the operation and customer satisfaction.

Railways started by concentrating on planning systems. A high degree of sophistication has been achieved, enabling the production of efficient plans, in the areas of timetable [1,2], rolling stock [3,4], and human resources [5-7]. 
Despite the advancements in planning tools, traffic disturbances are still currently handled by human dispatchers that take decisions regarding the solution of problems originated by unexpected events that occur during the course of operation. This work is always done under pressure, many times without leaving room for decision makers to fully study alternative solutions. The wide spread of communications enables real-time availability of information about the state of execution of the operation. Associated with real-time communication, new kinds of software products are emerging that provide effective support to railway dispatchers.

This paper discusses a real-time dispatching product that enables real-time monitoring of what is happening during the operation and provides optimizers to handle operational disruptions. At this moment, the product only presents solutions to crew-related problems, although it is being extended to handle rolling stockrelated problems. Scenario-based functionality to address major disruptions also is under development. Systems based on this product are in use in Dutch Railways, Finnish Railways, Norwegian Railways, and Danish Railways.

\section{Problem description}

Although we focus on dispatching, it is important to understand the whole planning and management process in a railway company, involving all phases and resources, and how dispatching fits in it.

\subsection{Planning phases}

Planning is the process of predicting the best use of available resources (track, rolling stock, and staff occupation) in order to provide a good service to customers, at the lowest possible cost. Planning aims at reaching an optimized solution, a solution with the minimum number of resources, thus a solution that achieves the highest productivity. However, since during the course of the operation there are likely to be deviations from the plan. If the plan is highly optimized there is the risk of introducing operational disruptions. It is, thus, preferable to sacrifice optimality for robustness, producing a plan that is prepared to cope with small deviations to the operation, obtaining the right balance between optimization and robustness.

Planning is subject to global and individual constraints that mutually interact, making it a very complex process. In order to control its complexity, railway companies divide planning in different phases and different types of resources (track and time, rolling stock, and crew), resulting in a set of smaller, and therefore less complex, subproblems (Figure 1).

The long-term planning phase deals with the regularity involved in the operation, leaving exceptions and irregularities to be handled later. This phase produces plans, without taking in account named resources (but rather abstract resources described in terms of important characteristics) and particular dates (but abstract week frequencies that capture the regularity of the operation). The longterm plan for track occupation corresponds to the timetable; the long-term plan 


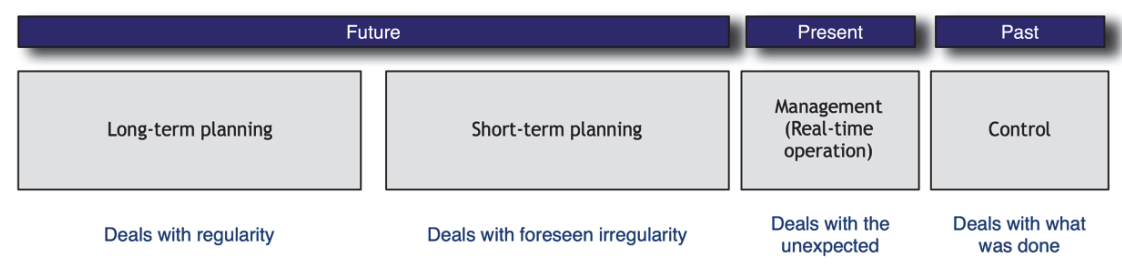

Figure 1: Planning and management phases.

for the rolling stock is composed of a set of rolling stock services and, eventually, rolling stock rosters; the long-term plan for the crew is composed of the crew duties and rosters [5].

In the next phase, resource allocation, named resources (both rolling stock units and crew members) are associated to the plans of the previous phase. The result of this phase is a daily plan for a certain period. This plan deals with named resources and calendar dates. For each named resource, the plans indicates the sequence of tasks that the resource has to perform during the period.

The short-term planning phase deals with the predicted irregularity involved in the operation. This phase modifies the daily plan taking into account (1) resource unavailability (rolling stock maintenance and planned absences of staff) and (2) timetable exceptions for particular dates (track work and special events), changing the rolling stock and crew plans accordingly. The short-term planning phase is done up to the day before the operation, when the plan becomes the responsibility of the dispatchers.

\subsection{Dispatching}

The dispatching phase deals with the unexpected events that occur during the day of operation. These events may be due to delays, breakdowns, or unexpected absence of crew.

The dispatching phase starts with the plan for the day, a timetable, a rolling stock plan, and a crew plan, and makes sure that the plan is executed as closely as possible to what was planned. The execution of the plan consists in making sure that the appropriate resources (rolling stock and crew) are in place when needed and complying, as much as possible, with the timetables of the trains. This involves monitoring of the plan as well as taking care of external conditions that affect operation. Whenever an expected condition occurs that has effect on the plan, dispatching performs re-planning, adapting the existing plan to the new reality. This is called plan recovery or just recovery. Recovery concerns rescheduling train timetables or reallocating resources to trains so that the operation can be performed as close as possible to the schedule.

Dispatching involves timetable (track occupation), rolling stock, and crew resources. Recovery, is further complicated due to the fact that different groups of dispatchers have the responsibility of recovery within each resource type. The 
decisions made by timetable dispatchers have impact on the decisions of rolling stock dispatchers, and the decisions of these on crew dispatchers. Sometimes, when a solution cannot be found downstream, some feedback must be given so that previous decisions upstream are revised. In other words, some concerted action must be taken between the three groups of dispatchers, in order to enable a good overall revised plan that takes all resources into account.

\section{CREWS real-time dispatcher}

The focus of this paper is the behavior of software product, CREWS Real-time Dispatcher (from now on, just RTD) that is part of a suite of products developed by SISCOG. This suite of products addresses all phases of the planning and management process, with a software module for each one. These modules interact with each other and are part of three main products: ONTIME, for the production of timetables; FLEET, for the production of the rolling stock plans; and CREWS, for the production of the crew plans.

\section{Handling small to medium-size disruptions}

Before the start of the operation, RTD loads the plan for the day. After this initial step, RTD has all the planned information for the day and is ready to start working. The human dispatchers are now responsible for manipulating that information and making the required changes to keep the plan updated with the real world while it changes.

The main communication with RTD is performed through messages that are sent from outside systems. This, together with the fact that only information about the day of the operation is handled, enables a high level of reactivity. The messages update the actual state of execution of the plan. They concern information about the actual departure and arrival of trains, rolling stock breakdowns, crew members that reported to work, and so on. RTD reacts to the messages by graphically displaying the current updated situation, highlighting existing and potential problems.

RTD is composed by two components, the monitoring and the rescheduling component.

\subsection{Monitoring component}

The monitoring component graphically represents the current situation of the plan and acts as a tool that enables dispatchers to consult the plan in search of solutions for existing problems. RTD gathers in real-time network-wide information about timetable, rolling stock, and crew. Whenever the system receives messages, it automatically updates the current state of the plan, verifying whether any constraint has been violated. It also propagates all changes to the affected entities in the plan. For example, a delay in a train may have effect on another train that will run a few hours later because the driver of the delayed train will not be able to 


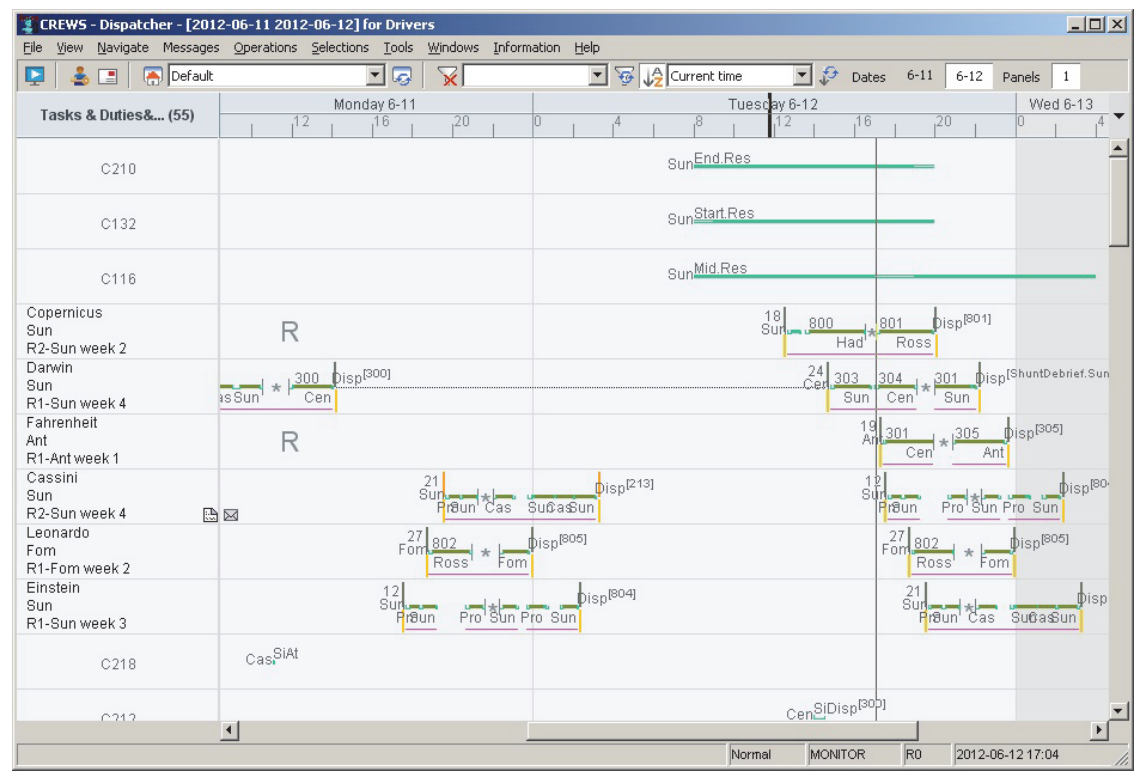

Figure 2: Sample of the monitoring screen.

operate the later train which he/she is planned to drive. When the system detects a violation, it signals it in the entities.

The monitoring component displays, for each resource in a Gantt chart, the sequence of tasks that are planned and a moving vertical line indicates the current time. As information is received, the monitoring component indicates, using different colors, the tasks that were completed, the tasks that are currently being performed, the tasks that are planned to be done without change, and the tasks that will deviate from the planned activities. Whenever there is a delay, the planned times and the actual expected times are shown as two different lines (Figure 2).

\subsection{Rescheduling component}

In a large number of cases, dispatchers have to react to outside conditions and reschedule some of the planned work in order to recover the normal operation. This is the role of the rescheduling component.

The rescheduling component helps deciding which problems should be solved, proposing actions for their solution. Problems can be solved by reassigning resources, using resources on reserve, or taking more drastic actions such as delaying or canceling trains.

When the rescheduling component is activated, the state of the operation is "frozen" in the rescheduling session, meaning that no messages are received in the session, and the dispatcher can study different scenarios to solve the problem. Rescheduling can be done manually or automatically (as described 
in [8]). In manual mode, the planner is in full control, taking all decisions, and the system validates the constraints. In automatic mode, the system takes most decisions, using an optimizer, the IP-Solver, a modified version of LUCIA (an integer programming algorithm [6] that alternates between generation, deletion, and optimization phases), where each duty is regarded as a modified version of a duty in the original plan, as described in [9].

The human dispatcher must define the problem to be solved. He/she starts by selecting the tasks that are to be re-planned and the places where the tasks can be planned. He/she may signal tasks that are not to be considered, marking tasks to be excluded from the automatic mode. Once the problem is defined, the human dispatcher asks IP-Solver to solve it. During the problem-solving process, a large number of potential duties are generated based on the duty templates, so that only duties that are interesting for replacing the existing duties are generated [10]. The human dispatcher may run different simulation to solve the problem. The rescheduling component uses a powerful optimizer, called JIT (JustIn-Time) Rescheduling, based on the work of [11]. It consists of a duty-scheduling problem solving method, specifically developed with the goal of returning effective solutions in the shortest possible amount of time.

\section{Handling major disruptions}

When a major disturbance occurs, either the problem is solved very quickly or the disruption may propagate to the entire network. It is important to find a solution that minimizes the impact on the operation.

To handle major disruptions, a scenario-based functionality is being incorporated in RTD. Scenarios are contingency plans that have been studied in advance and that can provide an answer to the problem at hand. A scenario is a description of a specific case of disruption, together with a temporary solution to handle the problem during a certain time interval. The solution describes the changes to be introduced in the timetable of trains that use the line section, as well as the changes in the corresponding rolling stock plans. Since RTD only addresses dispatching for crew members, scenarios correspond to a fast way to introduce alternative solutions for timetables and rolling stock. However, scenario-based functionality will also be useful when the automatic dispatching components are in place for timetable and rolling stock, since scenarios correspond to studied answers to certain emergency situations. In Europe, where the infrastructure management is separated from the operation, there will always be two entities evolved in the dispatching phase, the infrastructure provider and the operator. The infrastructure provider is responsible for decisions regarding the timetable and the operator is responsible for decision related to rolling stock and crew.

We discuss a scenario for the case of the Dutch Railways regarding a situation where a line blockage occurs between stations Hvsp and Hor, a line in the center of the Netherlands, blocking the train circulation in that stretch of the network. Since rolling stock needs certain railway facilities where compositions can turn around, it is necessary to choose stations that have such facilities. for these reasons, a line 


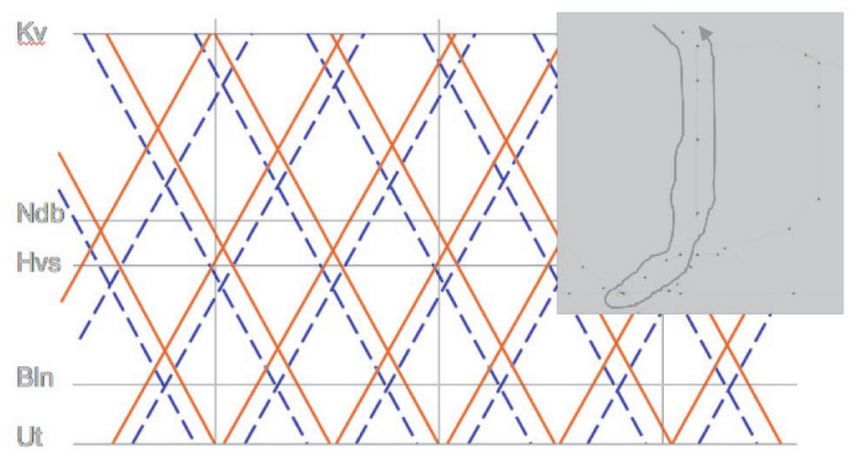

Figure 3: Kv-Ut time-space diagram.

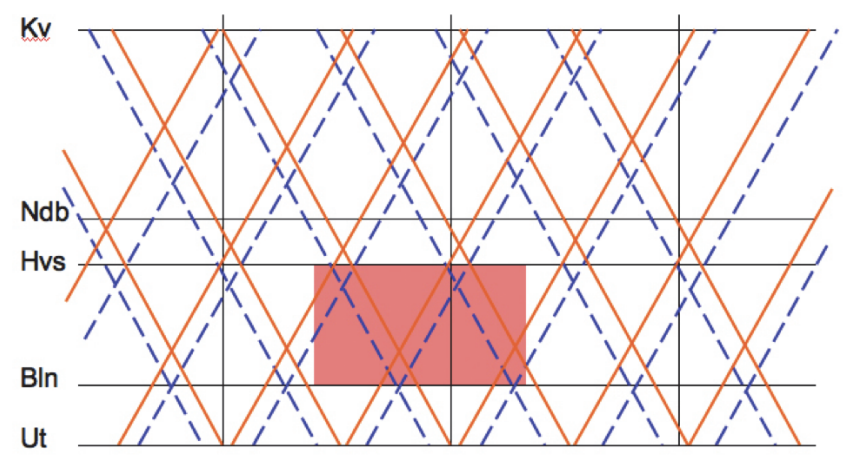

Figure 4: Line section Hvs-BIn with the time interval during which the blockage is expected to occur.

section larger than the one blocked by the accident usually ends up being closed, in this case between Hvs and Bln. There are two train series - a train series is a series of trains with common characteristics, that run between two stations, at regular intervals (eg. every hour or every half an hour) during the day - 4900 and 5700, running between $\mathrm{Kv}$ and Ut, the line to which the line section Hvs-BIn belongs, represented in the time-space diagram of Figure 3 as dashed and solid lines, respectively. The routes of these train series go past the section represented in Figure 3, but we only need to consider the line sections around the closed sections in order to handle the disruption. Since the line section Hvs-BIn cannot be used for a certain number of hours, we show a rectangle the space (line section) and time (time interval) that is blocked to the operation (Figure 4).

Figure 5 shows the solution that was designed for the scenario. Train series 4900 and 5700 are cancelled between Hvs and Bln (canceled segments are represented by dotted lines). A solution must then be found for the operation on the line sections on each side of the blocked section. All trains that cross the rectangle 


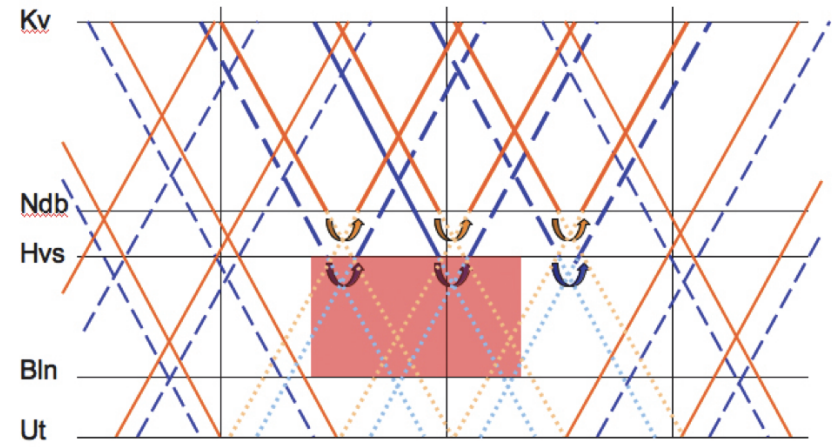

Figure 5: During the blockage, train series are turned in the north and cancelled in the closed sections and in the south.

must be cancelled or have their rolling stock handled in a different way in these line sections. On the North side (top part of Figure 5), trains that cross the rectangle, represented as heavy lines, are rescheduled. The 4900 series trains are turned on Hvs and the 5700 series trains on Ndb. On the South side (bottom part of Figure 5), a different solution is adopted, canceling the trains between Ut and BIn.

If during the operation, both tracks between Bln and Hvs become blocked for some reason, the dispatcher can request the RTD to instantiate this scenario for an estimated duration. RTD, then validates the crew duties against the new situation. Once the system has identified the parts of the plan that need to be changed, it runs a rescheduling algorithm to find a solution to handle the disruption. Figure 6 shows the solution duties (in a grey background) and compares them with the original duties (in a black background). The tasks that were changed are marked with a dark blue background, for easy identification by the user.

In spite of the speed increase in finding a solution due to the use of scenarios, there is still a time lag between the occurrence of an accident and the production of a plan to handle the situation. This reality requires a process plan composed of several phases (Figure 7). When a track blockage occurs, the normal plan is being executed but can no longer continue. While the dispatcher prepares a new plan in RTD, some ad-hoc plan must be followed to handle the immediate situation. At this point, some drastic decisions may be taken, e.g., the cancellation or delay of a train, due to the absence of a better plan. When a new plan is produced by RTD and is ready to be executed (that is all shareholders have been warned and have accepted it), it starts being executed. At that time, dispatchers must start preparing the start-up plan, that is the plan for making the transition between the new plan and the normal plan and that must start when the track is clear. When conditions are ready for the normal plan, the operation goes back to normal. 


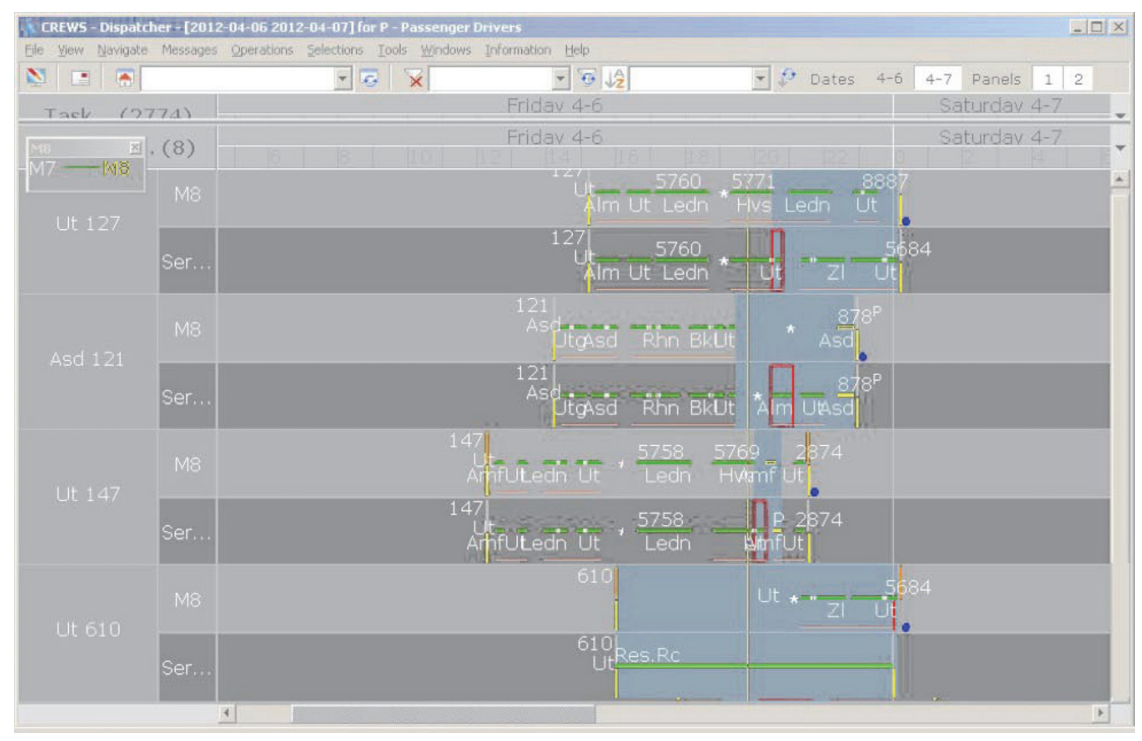

Figure 6: Automatic solution, showing the resulting and the original duties.

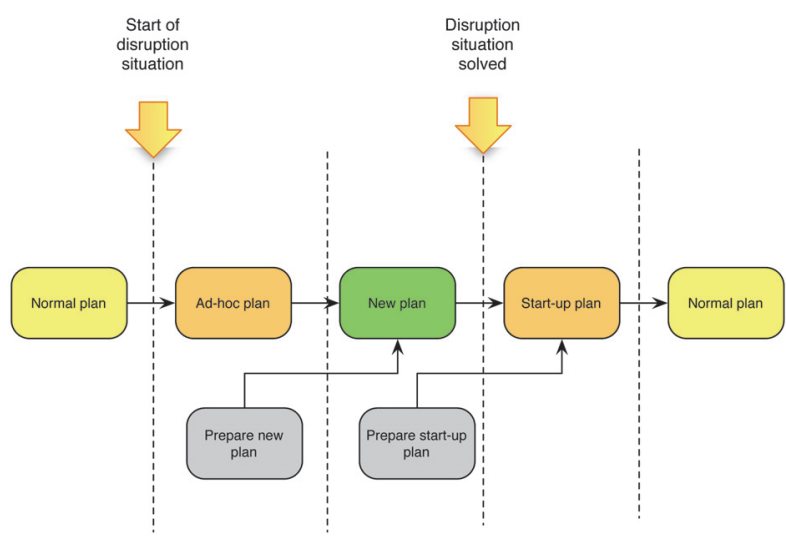

Figure 7: Phases in handling major disruptions.

\section{Conclusions}

Dispatching work is always done under time pressure, often without leaving room for decision makers to fully study alternative solutions.

This paper presents a real-time dispatching product that has been developed by SISCOG and that has proved to provide effective support to dispatchers in handling critical situations and enabling them to study alternative solutions in due time. 
The product enables real-time monitoring of the operation and provides optimizers to handle small to medium-size disruptions. The work on the dispatching component is being extended to handle rolling stock changes. Scenario-based functionality is also being developed to address major disruptions. In Europe, where the infrastructure management is separated from the operation, there will always be two entities evolved in the dispatching phase, the infrastructure provider and the operator. The infrastructure provider is responsible for decisions regarding the timetable and the operator is responsible for decision related to rolling stock and crew.

As communications evolve and are widely spread in railways, we expect realtime availability of information and more and more timely and accurate knowledge about the state of the operation. With the availability of a dispatching system, like CREWS RTD, we can expect to take effective use of that knowledge, with different levels of decision-support. Consequently, we can expect a high effectiveness and efficiency in handling different degrees of operation disruptions in complex transportation systems, and capable of replanning not only crew but also rolling stock and trains.

\section{Acknowledgements}

We would like to thank NSR, Erwin Abbink, Dennis Huisman, and Daniel Potthof for providing knowledge, insights, data and other information, fundamental for the understanding of the problem by SISCOG. We would also like to thank the CREWS team the work on the CREWS product and on CREWS optimizers.

\section{References}

[1] Cacchiani, V., Caprara, A. and Toth, P., A column generation approach to train timetabling on a corridor. Operations Research, 6(2), pp. 125-142, 2008.

[2] Lusby, R.M., Larsen, J., Ehrgott, M. and Ryan, D., Railway track allocation: models and methods. OR Spektrum, 31(4), pp. 1-41, 2009.

[3] Budai, G., Maróti, G., Dekker, R., Huisman, D. and Kroon, L., Rescheduling in passenger railways: the rolling stock rebalancing problem. Journal of Scheduling, 13(3), pp. 281-297, 2009.

[4] Vaidyanathan, B., Ahuja, R.K. and Orlin, J.B., Real-life locomotive planning: New formulations and computational results. Transportation Research Part B: Methodological, 42(2), pp. 147- 168, 2008.

[5] Martins, J.P. and Morgado, E., Case Studies in Planning Railroad Crew members. Proc. of 2010 Joint Rail conference, ASME, volume 2, pp. 535$544,2010$. 
[6] Abbink, E.J.W., Albino, L., Dollevoet, T., Huisman, D., Roussado, J. and Saldanha, R.L., Solving large scale crew scheduling problems in practice. Journal Public Transport, 3(2), pp. 149-164, 2011.

[7] Morgado, F., Saldanha, R.L., Roussado, J., Albino, L., Morgado, E. and Martins, J.P., Using AI local search to improve an OR optimizer. Proc. of the Annual Conference on Innovative Applications of Artificial Intelligence (IAAI-12), AAAI, 2012.

[8] Morgado, E. and Martins, J.P., CREWS_NS: Scheduling train crew in The Netherlands. Proc. of the Annual Conference on Innovative Applications of Artificial Intelligence (IAAI-97), AAAI, pp. 893-902, 1997.

[9] Huisman, D., Integrated and Dynamic Vehicle and Crew Scheduling. Ph.D. thesis, Erasmus University Roterdam, 2004.

[10] Morgado, E. and Martins, J.P., Automated Real-time Dispatching Support. Proc. of the 2012 APTA Rail Conference, 2012.

[11] Potthoff, D., Railway Crew Rescheduling - Novel Approaches and Extensions. Ph.D. thesis, Erasmus University Rotterdam, 2010. 